\title{
A Convex Gradient Coil Design for Vertical Field Open MRI

\author{
S.Y. Lee ${ }^{1}$, M.H. Cho ${ }^{2}$, C.H. Moon ${ }^{3}$, and H.W. Park ${ }^{4}$
}

\begin{abstract}
We introduce a convex gradient coil that can be used for interventional studies using a vertical field open MRI system. By placing gradient coil wires on two coaxial convex surfaces seeing each other, we can improve the accessibility to the patient during the scan, while maintaining the space for imaging. We have defined convex surfaces on hyperboloids at the prolate spheroidal coordinate. If we take account of accessibility at the figure of merit for the gradient coil performance, the convex gradient coil has better performance than conventional planar gradient coils.
\end{abstract}

Key words - Convex gradient coil, magnetic resonance imaging, interventional studies, prolate spheroidal coordinate

\section{INTRODUCTION}

Planar gradient coils have been widely used at vertical field MRI. As interventional applications using a vertical field MRI increase, it becomes very desired to have open structure at the design of a main magnet and gradient coils. Many research groups have developed design methods of open gradient coils. For the interventional studies using an open MRI, large gradient field strength is strongly desired while maintaining easy accessibility to the patient during the scan. The most efficient way to increase the gradient field strength is to reduce the gap size of the planar gradient coil. Reduction of the gap size is, however, very undesirable since small gap size will make it very difficult to perform interventional studies during the scan. In this study, we propose a new convex gradient coil that can be used in a vertical field MRI system. Utilizing convex gradient coil surfaces, we can increase the gradient field strength at the volume of interest while maintaining the openness at the entrance of the gradient coil. We have defined the convex gradient coil surfaces on hyperboloids at the prolate spheroidal coordinate with which we can control the curvature of the convex surface in an easy manner. We present various simulation results of the gradient coil performance with respect to the curvature of the convex surface.

\section{METHODS}

We have used the prolate spheroidal coordinate to define the convex gradient coil surface. The prolate spheroidal coordinate $(\alpha, \beta, \phi)$ is related by the cartesian coordinate $(\mathrm{x}, \mathrm{y}, \mathrm{z})$ as follows,

$$
\begin{aligned}
& x=a \sinh \beta \sin \alpha \cos \phi, \\
& y=a \sinh \beta \sin \alpha \sin \phi, \\
& z=a \cosh \beta \cos \alpha,
\end{aligned}
$$

where constant $\alpha(0 \leq \alpha \leq \pi)$ makes a convex hyperboloidal surface. The surface defined by $\alpha=0$, or $\pi$ is a needle-shaped surface, and the surface defined by $\alpha=\pi / 2$ is a flat plane. The surface with other $\alpha$ value is a hyperboloid that can be used as a convex gradient coil surface. An example of the convex hyperboloidal surface is shown in figure 1 .

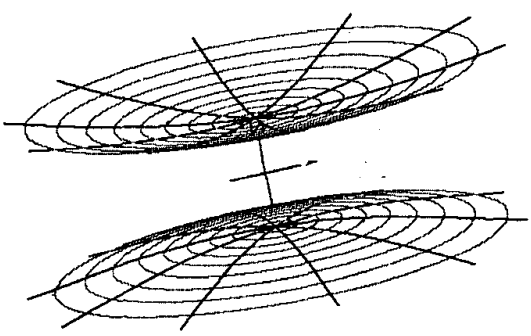

Fig.1 Convex gradient coil surface when $\alpha=1.3$

On the convex hyperboloidal surface, we have made fine triangular meshes to compute desired current density function. The desired current density function has been determined by iterative procedures[1]. After formulating an equation relating the magnetic field strength and the current density inside the triangular mesh element, we have defined a cost function as follows,

$f(\Sigma)=w_{W} W+\sum_{t=1}^{T} w_{t}\left[B_{z}\left(x_{t}, y_{t}, z_{t}\right)-B_{t}\right]^{2}$

where $\sum$ is the matrix representing the nodal current densities, $W$ is the magnetic energy stored by the current, $B_{t}$ is the desired magnetic field strength at given points, $B_{z}$ is the actual magnetic field strength, and $w_{W}, w_{t}$ are weighting factors. The nodal current density matrix $\sum$ has been found by minimizing the cost function using the conjugate gradient method. 


\section{RESULTS}

Various gradient coils have been designed with varing the curvature of the convex surface. The projection of the convex surface was chosen to be a circle so that it can be easily mounted on the pole piece of a permanent magnet. The radius of the projection of the gradient coil surface is $0.5 \mathrm{~m}$. The gap size at the entrance of the gradient coil is $0.5 \mathrm{~m}$. By varying $\alpha$ values, we can control the gap size at the center of gradient coil. At table 1, we show the gap sizes at the center of gradient coil with respect to $\alpha$ values.

Table 1. The gap size at the center of gradient coil (The gap size at the edge of gradient coil is $0.5 \mathrm{~m}$ )

\begin{tabular}{|c|c|c|c|c|}
\hline$\alpha$ & 1.5 & 1.4 & 1.3 & 1.25 \\
\hline $\begin{array}{c}\text { Gap size at } \\
\text { the center(m) }\end{array}$ & 0.49 & 0.47 & 0.41 & 0.37 \\
\hline
\end{tabular}

Using the conjugate gradient method, we have found minimum-inductance current density values at the node points. After obtaining an optimum current density function, we have determined coil wire patterns of transverse and longitudinal gradient coils. Coil wire patterns of the transverse gradient coil is shown in fig. 2. Using the coil wire pattern information, we have evaluated gradient coil performances, including gradient field linearity, gradient field strength, coil inductance and accessibity to the patient. The accessibility was defined by the ratio between the gap sizes at the entrance and center of the gradient coil. The performances of transverse gradient coil are shown in table 2.

Table 2. Performances of the transverse convex gradient coil

\begin{tabular}{|c|c|c|c|c|}
\hline$\alpha$ & 1.3 & 1.35 & 1.4 & 1.5 \\
\hline $\begin{array}{c}\text { Gap size at the } \\
\text { center (m) }\end{array}$ & 0.41 & 0.45 & 0.47 & 0.5 \\
\hline $\begin{array}{c}\text { Inductance } \\
(\mathbf{m H})\end{array}$ & 123.4 & 142.1 & 157.8 & 169.4 \\
\hline $\begin{array}{c}\text { Linearity error } \\
\left(\times \mathbf{1 0}^{-5}\right)\end{array}$ & 25.6 & 14.7 & 10.0 & 6.66 \\
\hline $\begin{array}{c}\text { Grad. strength } \\
(\mathbf{m T} / \mathbf{m} / \mathbf{A})\end{array}$ & 0.17 & 0.15 & 0.13 & 0.12 \\
\hline
\end{tabular}

As the gap size at the center decreases, gradient field strength and coil inductance decrease as can be expected. However, the gradient linearity error increases owing to reduced gap size. Performances of the longitudinal gradient coil are quite similar to those of the transverse gradient coil.

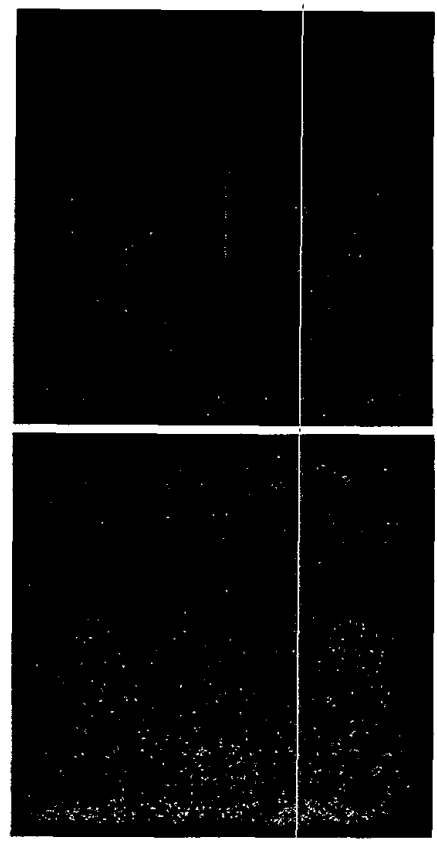

Fig. 2 Transverse gradient coil patterns. (upper: $\alpha=1.30$, lower: $\alpha=1.35$ )

\section{CONCLUSIONS}

It is expected that the proposed convex gradient coil can be greatly utilized for interventional applications using a vertical open MRI.

\section{References}

1. S. Pissanetzky, Meas. Sci. Technol. Vo1.3, pp.667673439,1992

'Dept. of Medical Engineering, Graduate School of East-West Medicine, Kyunghee University, Korea sylee01@nms.kyunghee.ac.kr

${ }^{2}$ Dept. of Medical Engineering, Graduate School of East-West. Medicine, Kyunghee University, Korea

${ }^{3}$ Dept. of Electrical Engineering, KAIST, Korea xmoon@athena.kaist.ac.kr

${ }^{4}$ Dept. of Electrical Engineering, KAIST, Korea hwpark@athena.kaist.ac.kr 